\title{
Recrystallization and Micronization of 4-Dimethylaminoantipyrine Using the Rapid Expansion of Supercritical Solution (RESS) Process
}

\author{
Chai-Siah Lee, Muoi Tang, Sheau-Ling Ho, and Yan-Ping Chen
}

\begin{abstract}
The rapid expansion of supercritical solution (RESS) process was successfully applied in this study for the recrystallization and micronization of an active pharmaceutical ingredient (API) of 4-dimethylaminoantipyrine. The untreated 4-dimethylaminoantipyrine had a large mean particle size with very wide particle size distribution ranging from 22 to $2468 \mu \mathrm{m}$. After the RESS treatment, the mean particle size of this API was significantly reduced to the desirable range of 1 to $5 \mu \mathrm{m}$. Furthermore, more uniform and much narrower particle size distribution was obtained after the RESS process. It was observed that the variation of the pre-expansion and post-expansion temperatures has significant effect on the mean particle size, particle size distribution and crystal habit. The DSC and XRD analyses results showed that there was polymorph transformation during the RESS process. The dissolution rate measurements had been conducted for the original and RESS treated APIs. The results demonstrated that higher dissolution efficiency was obtained as a consequence of significant particle size reduction.
\end{abstract}

Index Terms-Micronization, ress, 4-dimethylaminoantipyrine, polymorph.

\section{INTRODUCTION}

Many discovered pharmaceutical compounds have poor solubility in aqueous media and thus affect their dissolution rates and pharmaceutical effect in the gastrointestinal tract. Therefore, investigation of alternative strategies that can improve the therapeutic performance of pharmaceutical compound in human biological systems is of primary importance in pharmaceutical industry.

Many conventional particle size reduction techniques are constrained by their drawbacks. For example, grinding (milling) and mechanical crushing are limited by heat or mechanical degradation of pharmaceutical compounds. In order to overcome most of the drawbacks of the traditional micronization methods, supercritical fluid technology emerged as a new and interesting route for particle formation and it has been explored with great interest over the past decades [1]-[5]. It is regarded as a green technology and shows its greatest benefit of being environmentally benign. Supercritical carbon dioxide has received considerable attention by the pharmaceutical industry, in particular for pulmonary delivery, due to its mild critical temperature and

Manuscript received October 30, 2014; revised March 20, 2015.

Chai-Siah Lee and Yan-Ping Chen are with the Department of Chemical Engineering, National Taiwan University, Taipei, Taiwan (email: ypchen@ntu.edu.tw).

Muoi Tang and Sheau-Ling Ho are with the Department of Chemical and Materials Engineering, Chinese Culture University, Taipei, Taiwan. pressure. It poses no threat to the environment or human health due to its nontoxic, nonflammable and easily recycled properties.

The Rapid Expansion of Supercritical Solutions (RESS) process is used for compounds with high solubility in supercritical $\mathrm{CO}_{2}$. In this study, the RESS process was studied for the recrystallization and micronization of an active pharmaceutical ingredient (API) of 4-dimethyl-amino-anti-pyrine. 4-dimethylaminoantipyrine is a non-steroidal anti-inflammatory drug (NSAID) that is most commonly used with their analgesic, antipyretic, antirheumatic and anti-inflammatory properties to reduce or relieve pain, fever, inflammation and rheumatic [6], [7]. In this study, experimental parameters including the pre-expansion temperature, post-expansion temperature and nozzle diameter were varied in order to obtain the optimal operation conditions for the RESS process. The particle size, morphology, thermal behavior, crystal habit, and polymorphic behavior were analyzed to explore the performance of RESS process under different experimental conditions. Finally, the dissolution profiles before and after the RESS process for this API were conducted to compare the effect of particle size reduction.

\section{EXPERIMENTAL}

\section{A. Materials}

4-Dimethylaminoantipyrine $\left(\mathrm{C}_{13} \mathrm{H}_{17} \mathrm{~N}_{3} \mathrm{O}\right)$ was purchased from Sigma-Aldrich Co. (Taiwan) with purity greater than 99 mass\%. Carbon dioxide was used as the solvent in the RESS process and was supplied by Liu-Hsiang Gas Co. (Taiwan) with certified purity greater than 99 mass\%. All chemicals and solvents were of the purest grade available and used without any further purification.

\section{B. Experimental Setup and Procedures}

The similar experimental setup and procedures were used as presented in our previous literature [8]. The setup is mainly comprised of three main portions: supercritical carbon dioxide delivery, solute extraction, and particles precipitation.

Carbon dioxide was first supplied from a $\mathrm{CO}_{2}$ gas tank and then passed through a molecular sieves filter to remove any residual that might be present in the gas. Afterward, the solvent $\mathrm{CO}_{2}$ was liquefied to $275 \mathrm{~K}$ using a cooler and then compressed to the desired pressure by means of a HPLC pump. The desired pressure of $\mathrm{CO}_{2}$ was controlled by a manually operated back pressure regulator. The pressurized 
$\mathrm{CO}_{2}$ was passed through pre-heated circulating coils where $\mathrm{CO}_{2}$ was heated to the supercritical condition.

The extraction column $\left(75 \mathrm{~cm}^{3}\right.$ internal volume $)$ was loaded with $10 \mathrm{~g}$ of API solute and packed by alternate layers of glass beads with $0.5-0.6 \mathrm{~mm}$ diameter to increase the contact area between the API particles and the supercritical $\mathrm{CO}_{2}$. The column ends were plugged with $0.5 \mu \mathrm{m}$ stainless filter to avoid any solute carry over with the $\mathrm{CO}_{2}$ flow. Then, the heating jacket was applied on the column and was heated to the studied temperature. The extraction temperature (318 $\mathrm{K})$ and pressure (200 bar) were measured through thermocouple and pressure transducer. Once the extraction temperature and pressure were achieved, the supercritical $\mathrm{CO}_{2}$ was introduced into the extraction column at constant flow rate in the extraction line. Then, the solute was kept under supercritical $\mathrm{CO}_{2}$ for an hour to ensure complete equilibrium state has been attained before expansion.

After the equilibrium was reached, the supercritical solution exited from column was assumed to be saturated with the solute. The supercritical solution was then led to the pre-expansion line which was heated through a heating tape. Pre-expansion temperature was set higher than the solute melting point in order to prevent premature particle precipitation inside the expansion line and nozzle tube during the expansion period. Subsequently, the solution was passed through a $5 \mathrm{~cm}$ capillary nozzle $(25$ or $50 \mu \mathrm{m}$ in diameter) and experienced a rapid expansion from supercritical condition to ambient state. Rapid phase change of $\mathrm{CO}_{2}$ from the supercritical state to gas state induced high supersaturation of the solute and thus resulted in the formation of very fine particles.

The precipitated particles were sprayed on a collecting vessel and the post-expansion temperature (temperature in the collecting vessel) was controlled by a water bath. The released $\mathrm{CO}_{2}$ was vented off through an exit on the top of the collecting vessel. The obtained samples were kept in a desiccator avoiding from light at room temperature prior to particles characterization. After each experiment, the expansion line was cleaned with acetone or ethanol to remove any residual particle which may have been left in the line.

The crystal habit of the RESS treated 4-dimethylaminoantipyrine was examined using scanning electron microscope (SEM, JEOL JSM-5600 or JEOL JSM-6335F). The mean particle size and particle size distribution (PSD) were calculated using image analysis software (Image J). At least 300 well-separated particles were arbitrarily selected from SEM photomicrographs for the statistical analysis to yield the average particle size and number average size distribution results. The crystal structures of the RESS processed API were analyzed using the X-ray diffractometer (XRD, MAC Science M03XHF) where the diffractograms have been collected in a $2 \theta$ range of $5-40^{\circ}$ with a scanning rate of $3 \%$ min. Thermal behavior of particles was investigated using the differential scanning calorimeter (Jade DSC, Perkin Elmer) at a heating rate of 5 $\mathrm{K} / \mathrm{min}$.

The dissolution analysis was carried out with a calibrated dissolution tester (Shin Kwang Machinery, DT3) using the paddle method that is ascertained by the United States Pharmacroscopeia [9]. A suitable dissolution medium with specified $\mathrm{pH}$ value was prepared and filled into a dissolution vessel. The temperature of the dissolution medium and the agitator speed were maintained at $310 \mathrm{~K}$ and $50 \mathrm{rpm}$ respectively. Afterward, accurately weighted of solute (30-50 $\mathrm{mg}$ ) was added to the dissolution medium $(800 \mathrm{~mL})$. At certain time intervals, each specimen (3-5 mL) was withdrawn and was filtered through a syringe filter (nylon, $0.45 \mu \mathrm{m}$ pore size). Finally, the UV/vis spectrophotometer, (Shimadzu, TCC-240A) was used to determine the concentration of the dissolved amount of solute in a withdrawn sample at specified wavelength.

\section{RESUlTS AND DISSCUSSION}

In this study, RESS process was applied for the recrystallization and micronization of 4-dimethylaminoantipyrine (4-DAA). The influence of various operating parameters (pre-expansion temperature $T_{\text {pre, }}$ post-expansion temperature $T_{\text {post }}$, and nozzle diameter $D_{n}$ ) on the particle size, size distribution and morphology was investigated. The original API of 4-DAA has very broad particle size distribution, ranging from 22 to $2468 \mu \mathrm{m}$. The original particle size was divided into two categories. The first category includes $83 \%$ of the total particles that has particle size less than $500 \mu \mathrm{m}$. The mean size of the first category is $132.12 \mu \mathrm{m}$. The second category includes $17 \%$ of the total particles that has particle size greater than $500 \mu \mathrm{m}$. The mean size of the second category is $1124.69 \mu \mathrm{m}$. Ten experimental runs have been conducted in our RESS study under various operation parameters. The resulting mean particle size $(M S)$ and its standard deviation $(S D)$, the crystal habits and the polymorph forms are presented in Table I.

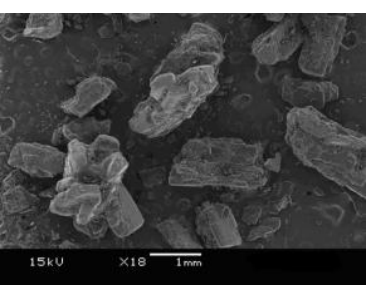

(a)

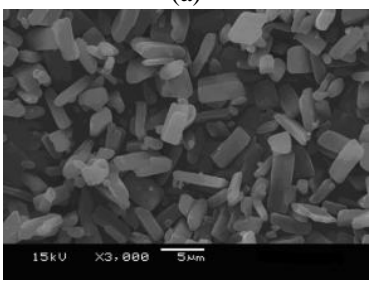

(b)

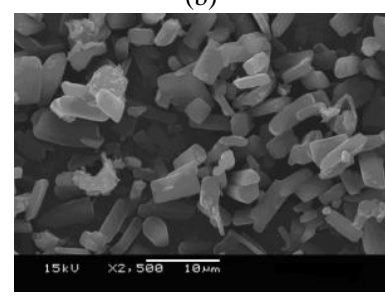

(c)

Fig. 1. Comparison of the SEM images (a) the original 4-DAA and (b) RESS treated 4-DAA from experimental run D8, (c) RESS treated 4-DAA from experimental run D9. 
After RESS treatment, the mean particle size of the drugs was significantly reduced to much smaller size, in the range of 1.92 to $7.64 \mu \mathrm{m}$, as shown in Table I. It is observed that experimental runs D2 and D3 yields comparably small particles with mean size around $2 \mu \mathrm{m}$. Experimental run D2, however, results in the narrowest size distribution with SD of
$1.41 \mu \mathrm{m}$. From our overall examination, experimental runs D8 and D9 yields small enough particles and the most uniform crystal habit. Fig. 1 shows the comparison of SEM images between the original and RESS treated 4-DAA (experimental runs D8 and D9).

TABLE I: THE EXPERIMENTAL RESULTS OF THE RESS TREATMENT OF 4-DAA UNDER VARIOUS OPERATION CONDITIONS

\begin{tabular}{|c|c|c|c|c|c|c|c|}
\hline Sample & $\begin{array}{l}T_{\text {pre }} \\
(\mathbf{K})\end{array}$ & $\begin{array}{l}T_{\text {post }} \\
(\mathbf{K})\end{array}$ & $\begin{array}{c}D n \\
(\mu \mathrm{m})\end{array}$ & $\begin{array}{c}M S \\
(\mu \mathrm{m})\end{array}$ & $\begin{array}{c}S D \\
(\mu \mathrm{m})\end{array}$ & Crystal Habit & $\begin{array}{c}\text { Polymorph } \\
\text { Form }\end{array}$ \\
\hline \multirow{2}{*}{ Original } & & & & $132.12(83 \%)$ & 94.98 & highly irregular and & I \\
\hline & & & & $1124.69(17 \%)$ & 489.19 & non-uniform & \\
\hline D1 & 373 & 293 & 50 & 7.64 & 5.78 & Irregular & II \\
\hline D2 & 383 & 293 & 50 & 2.15 & 1.41 & $\begin{array}{c}\text { rectangular and } \\
\text { rod-shaped }\end{array}$ & II \\
\hline D3 & 393 & 293 & 50 & 1.92 & 1.76 & $\begin{array}{l}\text { rectangular and } \\
\text { rod-shaped }\end{array}$ & II \\
\hline D4 & 383 & 283 & 50 & 4.77 & 2.72 & $\begin{array}{l}\text { rectangular and } \\
\text { needle-like }\end{array}$ & II \\
\hline D5 & 383 & 303 & 50 & 5.40 & 3.62 & irregular & II \\
\hline D6 & 373 & 293 & 25 & 5.37 & 3.67 & irregular & II \\
\hline D7 & 383 & 293 & 25 & 3.73 & 1.48 & $\begin{array}{l}\text { rectangular and } \\
\text { rod-shaped }\end{array}$ & II \\
\hline D8 & 393 & 293 & 25 & 3.31 & 1.59 & $\begin{array}{l}\text { rectangular and } \\
\text { rod-shaped }\end{array}$ & II \\
\hline D9 & 383 & 283 & 25 & 3.83 & 1.94 & $\begin{array}{c}\text { rectangular and } \\
\text { rod-shaped }\end{array}$ & III \\
\hline D10 & 383 & 303 & 25 & 5.00 & 3.26 & irregular & II \\
\hline
\end{tabular}

The influence of pre-expansion temperature $\left(T_{\text {pre }}, 373\right.$, 383 and $393 \mathrm{~K}$ ) on the particle size was investigated under the fixed extraction conditions (experimental runs D1-D3, and D6-D8 in Table I). The mean particle size was greatly affected by variation of pre-expansion temperature for the capillary nozzle with different diameters. At higher pre-expansion temperature, smaller particle size was obtained. Similar findings were reported for the micronization of anthracene [10] and ibuprofen [1]. As a supercritical solution is heated before expansion, the resulting unsaturated solution would prevent the particle formation in the capillary nozzle. This allows shorter nucleation and growth time, and thus smaller particle size was obtained. As shown in Table I, the crystal habit was changed from the irregular shape to the mixed type of rectangular and rod-shaped particles with increasing pre-expansion temperature.

By increasing the post-expansion temperature $\left(T_{\text {post }}\right)$ from 283 to $293 \mathrm{~K}$ (experimental runs D4 and D2, or experimental runs D9 and D7 in Table I), the mean particle size was decreased for both sizes of nozzle diameters. This was affected by the difference between the pre-expansion and the post-expansion temperatures. The lower post-expansion temperature induced an early nucleation of API prior to expansion. This early nucleation would lead to the formation of larger API particles.

However, continuous increase of the post-expansion temperature from $293 \mathrm{~K}$ to $303 \mathrm{~K}$ would enlarge the mean particle size (experimental runs D2 and D5, or experimental runs D7 and D10 in Table I). This may be due to the influence of another competing factor. Higher post-expansion temperature may cause both lower supersaturation ratio and nucleation rate, and this resulted in the formation of larger particles. The crystal habits were also observed from the mixed type of rectangular and rod-shaped particles to irregular form as presented in Table I.

Two kinds of nozzle with different diameters $\left(D_{n}, 25\right.$ and $50 \mu \mathrm{m}$ ) were used to investigate its effect on the mean particle size and crystal habit. Examining the results in Table I, the influence of nozzle diameter is not clear and significant. It probably means that the diffusion manners of sprayed supercritical solution were almost the same within the nozzle diameter range of 25 and $50 \mu \mathrm{m}$. Variation of nozzle diameter in this study does not cause apparent effect on the crystal habits.

The XRD diffraction patterns obtained for the RESS processed 4-DAA showed differences with respect to the unprocessed API under the experimental conditions in this study. Fig. 2 shows the XRD results for the original 4-DAA, and the RESS treated 4-DAA from experimental runs D8 and D9 in Table I. The original 4-DAA has four major XRD peaks at $8.97^{\circ}, 20.65^{\circ}, 23.51^{\circ}$ and $29.03^{\circ}$ respectively. The original 4-DAA is identified as polymorph I. The characteristic major peaks for the RESS treated 4-DAA are 
located at $9.13^{\circ}, 13.89^{\circ}, 19.03^{\circ}, 22.89^{\circ}$ (polymorph II, from experimental run 8$) ; 8.83^{\circ}, 12.69^{\circ}, 20.25^{\circ}, 23.01^{\circ}$ (polymorph III, from experimental run 9), respectively. To our knowledge, the polymorph forms for 4-DAA are not reported in literature. The polymorph forms of RESS processed 4-DAA under various operation conditions are listed in Table I.

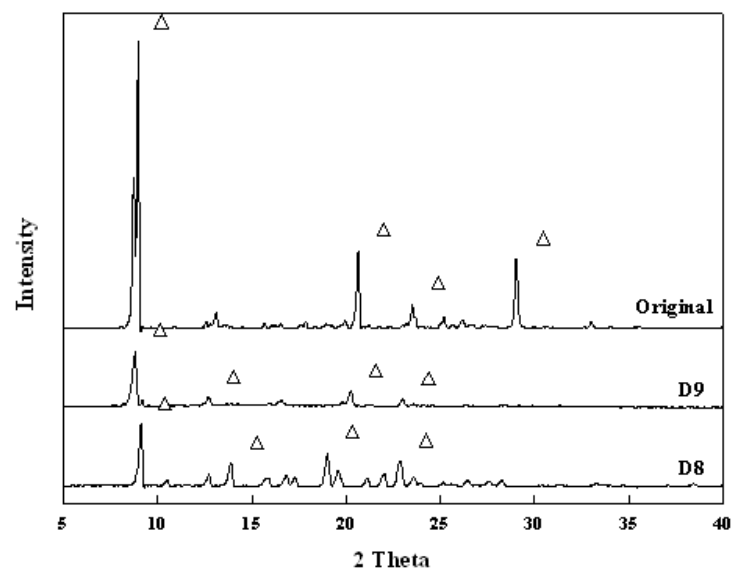

Fig. 2. The XRD results for (a) the original 4-DAA, (b) the RESS treated 4-DAA from experimental run D8, (c) the RESS treated 4-DAA from experimental run D9.

Different thermal behavior was observed between the original and processed 4-DAA. Fig. 3 presents the DSC analysis results for the original 4-DAA, and those from experimental runs D8 and D9 listed in Table I. The original 4-DAA (polymorph I) has its melting point at $382.18 \mathrm{~K}$ while the melting peak for the RESS treated 4-DAA (polymorph II, experimental run D8) was shifted to a lower temperature at $378.31 \mathrm{~K}$. The melting point for polymorph III 4-DAA (experimental run D9) was located at $378.22 \mathrm{~K}$. A decrease in the intensity of the endothermic peak for the RESS processed 4-DAA is observed. This result may probably be caused by the significant particle size reduction or loss of crystallinity after the process of recrystallization and micronization.

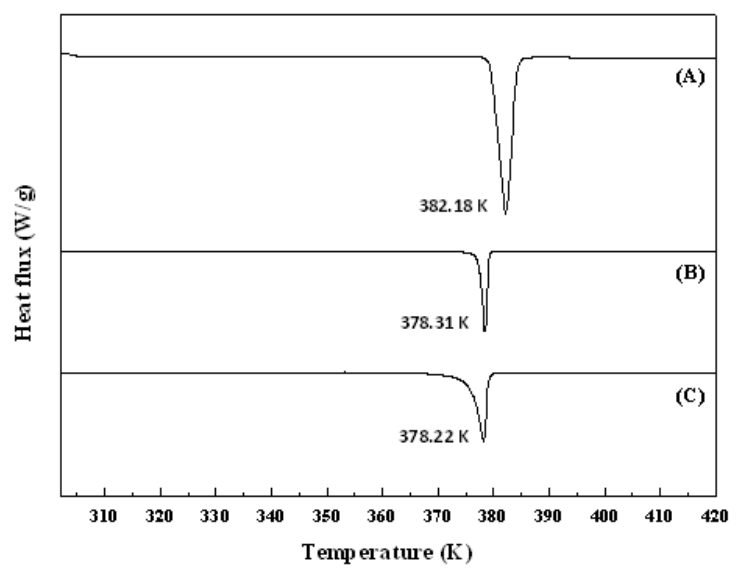

Fig. 3. The DSC analysis results for (a) the original 4-DAA, (b) experimental run D8, (c) experimental run D9.

A standard intestinal phosphate solution with $\mathrm{pH} 6.8$ was used as the dissolution medium for 4-DAA. The comparison of dissolution profiles between the original and the RESS-processed 4-DAA are presented in Fig. 4. The experimentally measured dissolution profile was correlated by a semi-empirical Weibull model [11]. The RESS process has effectively enhanced the dissolution performance by a factor of 2.99 for sample D9, 3.15 for sample D8 and 4.37 for sample D2 listed in Table I under various operation conditions.

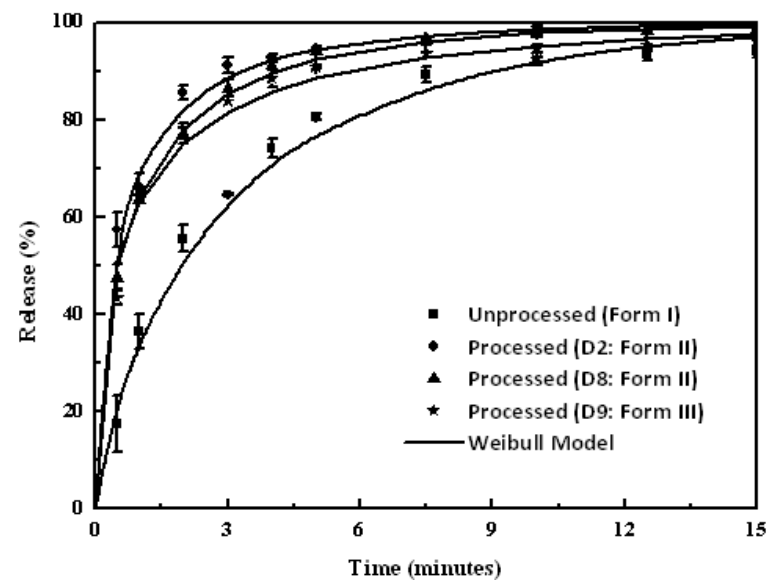

Fig. 4. The comparison of dissolution profiles between the original and the RESS-processed 4-DAA.

\section{CONCLUSIONS}

This study demonstrates that the RESS process successfully micronized the API of 4-DAA (4-dimethylaminoantipyrine). The original particle size of 4-DAA was very large, ranging from $22 \mu \mathrm{m}$ to $2468 \mu \mathrm{m}$. After RESS treatment, much smaller particles with mean size around $2 \mu \mathrm{m}$ was obtained. The RESS treated 4-DAA showed uniform morphology and much narrower size distribution. The experimental results revealed that the pre-expansion and post-expansion temperatures had apparent influence on the mean particle size, size distribution and crystal habit. The crystal structures of RESS processed 4-DAA indicated polymorph conversion. The XRD and DSC analyses show consistent results for various polymorph forms. The RESS treated 4-DAA shows an enhanced the dissolution performance up to 4.37 times.

\section{ACKNOWLEDGMENT}

The authors are grateful to the Ministry of Science and Technology, Taiwan, for supporting this research.

\section{REFERENCES}

[1] D. Kayrak, U. Akman, and Ö. Hortaçsu, "Micronization of ibuprofen by RESS,” J. Supercri. Fluids, vol. 26, pp. 17-31, 2003.

[2] Y. Hakuta, H. Hayashi, and K. Arai, "Fine particle formation using supercritical fluids," Current Opinion in Solid State and Materials Science, vol. 7, pp. 341-351, 2003.

[3] Z. Huang, G. B. Sun, Y. C. Chiew, and S. Kawi, "Formation of ultrafine aspirin particles through rapid expansion of supercritical solutions (RESS)," Powder Technology, vol. 160, pp. 127-134, 2005.

[4] J. Chingunpitak, S. Puttipipatkhachorn, S. Tozuka, K. Moribe, and K Yamamoto, "Micronization of dihydroartemisinin by rapid expansion of supercritical solutions," Drug Dev. Ind. Pharm., vol. 34, pp. 609-617, 2008.

[5] C. S. Su, M. Tang, and Y. P. Chen, "Micronization of nabumetone using the rapid expansion of supercritical solution (RESS) process," $J$ Supercri. Fluids, vol. 50, pp. 69-76, 2009. 
[6] Y. M. Chen and Y. P. Chen, "Measurements for the solid solubilities of antipyrine, 4-aminoantipyrine and 4-dimethylaminoantipyrine in supercritical carbon dioxide," Fluid Phase Equilib, vol. 282, pp. 82-87, 2009.

[7] D. Costa, A. Vieira, and E. Fernandes, "Dipyrone and aminopyrine are effective scavengers of reactive nitrogen species," Redox Report, vol. 11, pp. 136-142, 2006.

[8] P. C. Lin, C. S.u, M. Tang, and Y. P. Chen, "Micronization of ethosuximide using the rapid expansion of supercritical solution (RESS) process," J. Supercri. Fluids, vol. 72, pp. 84-89, 2012.

[9] M. D. Rockville, The United States Pharmacopeia, 32nd rev. ed., United States Pharmacopeial Convention, 2009.

[10] H. Kwak, J. Jung, S. Y. Bae, and H. Kumazawa, "Preparation of anthracene fine particles by rapid expansion of a supercritical solution process utilizing supercritical $\mathrm{CO}_{2}$," Korean J. Chem. Eng., vol. 21, pp. 1245-1249, 2004.
[11] P. Costa and J. M. S. Lobo, "Modeling and comparison of dissolution profiles," Eur. J. Pharm. Sci., vol. 13, pp. 123-133, 2001.

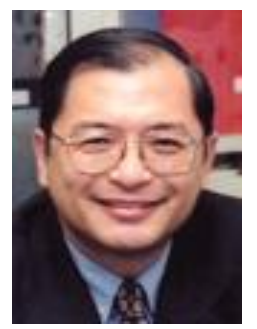

Yan-Ping Chen is a professor of chemical engineering at National Taiwan University, Taipei, Taiwan. He has his B.S. degree in chemical engineering from National Taiwan University in 1976, and his Ph. D. degree in chemical engineering from Rice University, Houston, Texas, USA in 1982. His major research interests include the thermodynamics of phase equilibrium, supercritical fluid technology, and fundamental investigations on gas hydrate systems. 